\title{
The biological properties of carotenoids
}

\author{
Norman I. Krinsky \\ Department of Biochemistry, Tufts University School of Medicine, Boston, MA 02111
}

\begin{abstract}
The biological properties of carotenoids can be divided into functions, which are essential to the well-being of organisms, and actions or associations, which are either responses to the administration of carotenoids, or are phenomena which still lack evidence of a causal relationship to the presence of carotenoids. Regardless of the strength of the evidence for these various biological properties, there must be either a physical or chemical basis that will serve to explain these phenomena. Many of the reported effects still require further investigations to help us understand the relationship between chemical properties and biological activity.
\end{abstract}

\section{INTRODUCTION}

There have been so many biological properties ascribed to carotenoids that Olson (ref. 1) suggested that they should be classified as either functions, actions or associations. Although he defined functions as properties essential for the normal well-being of the organism, the differences between actions and associations are not as clear. In this review, I will not try to differentiate between responses to the administration of carotenoids, or responses attributed to the presence of these compounds. What remains to be determined in whether there is a causal relationship between the presence of the carotenoid pigment and the biological property being described.

\section{PHYSICAL AND CHEMICAL PROPERTIES OF CAROTENOIDS}

Ultimately, the diverse biological effects attributed to carotenoids have to be explained in terms of their physical and chemical properties, many of which are shown in Fig. 1.

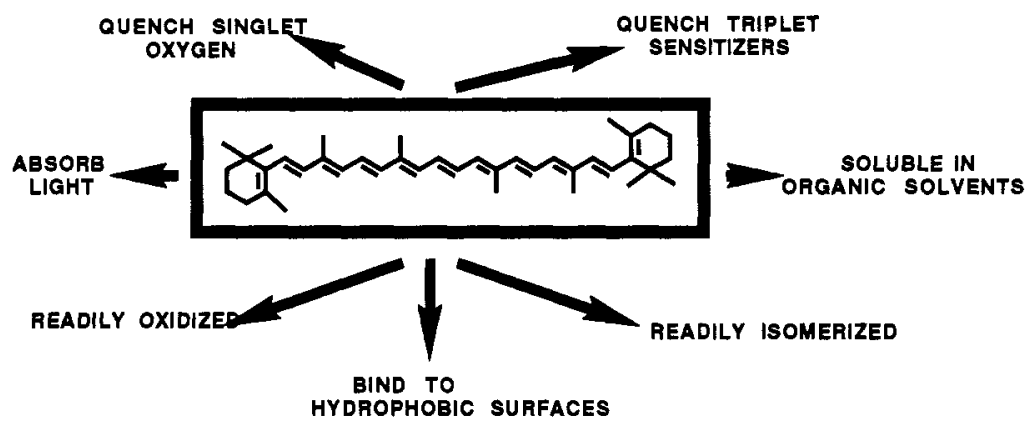

Fig. 1. Physical and chemical properties of carotenoids

We have learned a great deal about the physical properties of carotenoids with respect to their ability to absorb light and transfer radiant energy, from work in photosynthesis, but other properties have not been adequately related to biological effects. For example, the hydrophobic nature of most carotenoids defines where they should be found in biological systems, but most attention has been paid to their localization in the lipid environment of cells, and not enough to this same property being expressed as an interaction between carotenoids and proteins. This is not to minimize the important and elegant work carried out with the crustacean carotenoproteins (ref. 2), but to point out that we know very little about the nature of the interaction between carotenoids and the enzymes that metabolize them, and nothing at 
all about the proteins that define the localization of lutein and zeaxanthin in the macula of the primate retina (ref. 3,4$)$. Some of these physical and chemical properties are described below.

\section{Light absorption}

Probably the best known property of carotenoids is their ability to absorb light. We see evidence of this all through nature, ranging from many of the colors of plant material such as fruits and vegetables to the coloration of birds and invertebrates. In addition, many species contain brightly colored oil droplets associated with their retinal cones. The process of absorbing light involves the formation of the singlet state of carotenoids ( ${ }^{1}$ carotenoid), as shown below:

$$
\text { carotenoid + hv -----> } 1 \text { carotenoid }
$$

The singlet state, $S_{2}\left(B_{u}\right)$, is very short lived $(\tau=200 \mathrm{fs})$, and probably collapses to an $S_{1}\left(A_{g}\right)$ state with $\tau=20$ ps.

\section{Energy transfer}

In addition to absorbing light directly, carotenoids can also be excited by an energy transfer reaction to form the triplet state species ( ${ }^{3}$ carotenoid). The best known energy transfer reactions are the transfer of energy from a suitable triplet sensitizer to a carotenoid, or the transfer of excitation energy from singlet oxygen $\left({ }^{1} \mathrm{O}_{2}\right)$ to a carotenoid. In each case, as seen below, the ${ }^{3}$ carotenoid species is formed.

$$
\begin{aligned}
& { }^{3} \text { sen }+ \text { carotenoid }--->\text { sen }+{ }^{3} \text { carotenoid } \\
& { }^{1} \mathrm{O}_{2}+\text { carotenoid }--->{ }^{3} \text { carotenoid }+{ }^{3} \mathrm{O}_{2}
\end{aligned}
$$

As will be discussed below, the ability of carotenoids to accept energy from excited sensitizers has been demonstrated by the formation of ${ }^{3}$ carotenoid from ground state carotenoid and a sensitizer and light. The quenching of ${ }^{1} \mathrm{O}_{2}$ had been suggested by photochemists working with photosensitized systems, but it was not until 1968 that Foote first demonstrated this reaction between ${ }^{1} \mathrm{O}_{2}$ and $B$-carotene (ref. 5). The ability of carotenoids to quench ${ }^{1} \mathrm{O}_{2}$ is now widely accepted (ref. 6). Since the ability to quench is a function of the effective number of conjugated double bonds, it is not surprising that lycopene is a more effective ${ }^{1} \mathrm{O}_{2}$ quencher than is $\mathrm{B}$-carotene (ref. 7, 8).

\section{Chemical reactivity}

Carotenoids can undergo many reactions with a wide variety of chemical reagents, some of which might be similar to chemicals found in biological systems. The process that draws much attention in biological systems is the oxidation of carotenoids, during which time they may function as biological antioxidants. The ready ability of carotenoids to react with radical species has served as the basis for the determination of the activity of various lipoxygenases. The basis for the assay involves an oxidative interruption of the conjugated double bond system which is invariably accompanied by a loss of the visible absorption, or a "bleaching" of the carotenoid. Much of the earlier work has been reviewed recently (ref. 9, 10, 11), but some recent observations on the products of $\beta$-carotene autoxidation, radical-initiated oxidation, or the chemical reaction of carotenoids with ${ }^{1} \mathrm{O}_{2}$ indicate that we still have much to learn about secondary products of carotenoids other than retinoids (ref. 12,13, 14).

\section{Solubility}

Most of the carotenoid pigments present in nature are quite water-insoluble. As such, one would expect to find them associated with the hydrophobic environment in biological systems. Lipids are one class of hydrophobic compounds, but even proteins can have strong hydrophobic regions which can play an important role in protein-protein interactions, as well as in protein-ligand interactions. The specificity of the latter interaction can range from very high, as in the case of enzymes binding hydrophobic compounds tightly at catalytically active sites, to relatively low, as in the case of human serum albumin, which can bind a wide variety of hydrophobic compounds.

\section{Protein binding}

The best example of protein binding with $\beta$-carotene is the 15,15'-dioxygenase of intestinal and other tissues. This enzyme requires carotenoids with at least one unsubstituted $B$-ring which allows cleavage to form a family of $\mathrm{C}_{20}$ compounds known as retinoids (ref. 15). Unfortunately, little is known about the 
process because the enzyme has not been purified to the extent that it can be used as a reagent for mechanistic studies.

\section{Isomerization}

Although isomerization reactions of metabolites of carotenoids are extremely important in biological systems such as the visual system (ref. 16), we know very little about the isomerization of intact carotenoids. For example, cis-phytoene is a biosynthetic precursor of the all-trans isomers of carotenoids, but we do not know how or when or why the isomerization reactions take place. In some organisms, such as Dunaliella bardawil, the products of biosynthesis may consist of as much as $40-50 \%$ of cis-isomers but the reason for their appearance or the mechanism of formation is unclear (ref. 17). We also find different isomeric forms of carotenoids present in different portions of the photosynthetic apparatus, and Koyama has presented us with a rational explanation for the presence of these isomeric forms (ref. 18). However, we still have to learn how they are made, and targeted to specific cellular sites.

\section{BIOLOGICAL PROPERTIES OF CAROTENOIDS}

Some of the properties that have been attributed to carotenoids in biological systems are shown in Fig. 2 . In some cases, these properties can be considered to be biological functions, as they appear to be essential for the life of the organism. Other properties can be demonstrated but may not be essential to the system, and can be considered to be actions or associations of carotenoids. Many of the biological properties that have been associated with carotenoids are shown in Fig. 2

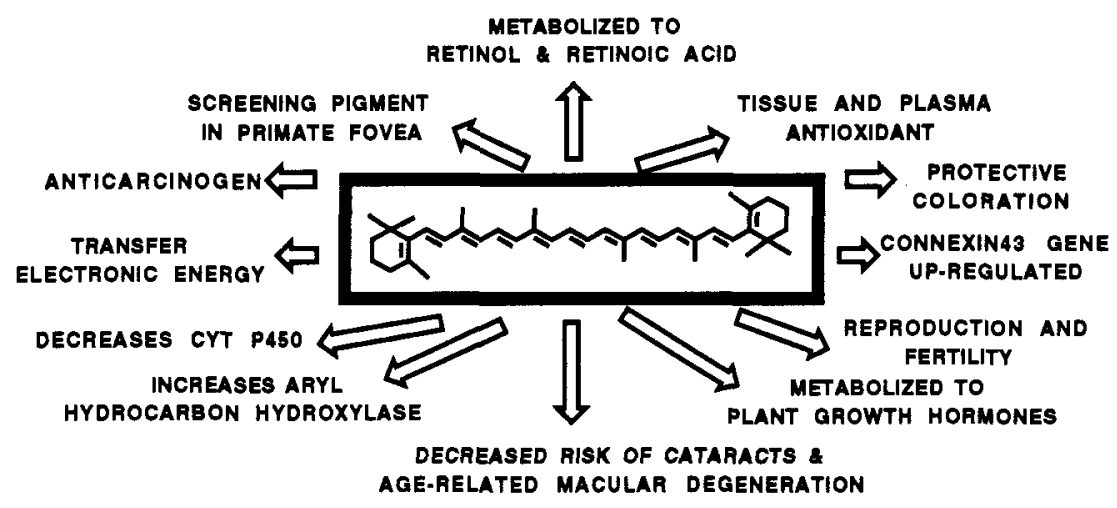

Fig. 2. Biological properties of carotenoids

\section{Biological Properties: Functions}

Using a definition of function that only considers properties essential to the well-being of organisms, we are limited to a very small number of examples, including energy transfer in photosynthesis, energy transfer in photoprotection, and metabolic conversion to retinoids in animals not ingesting an adequate amount of preformed vitamin $A$.

Energy transfer in photosynthesis. Much of the current material concerning this function has been reviewed in articles describing the role of carotenoids in photosynthetic organisms. The most recent description is that of Cogdell at this meeting (ref. 19). In essence, energy transfer involves the direct excitation of carotenoids by light to form the first excited singlet state $\left({ }^{1}\right.$ carotenoid), and the subsequent transfer of this excitation energy to a photoresponsive pigment, such as a chlorophyll, to initiate the process of photosynthesis.

$$
\begin{aligned}
& \text { carotenoid }+ \text { hv } \ldots . . .>1_{\text {carotenoid }}
\end{aligned}
$$

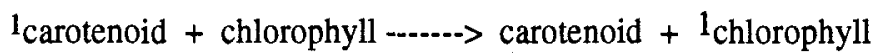

This type of process can effectively extend the wavelength of light available to an organism for photosynthesis, since the bulk of carotenoids present in photosynthesizing systems absorbs at wavelengths different form those of the photoactive chlorophylls or bacteriochlorophylls.

Energy transfer in photoprotection. Among the insults that biological systems are subjected to is the process of photodynamic action, or the damage caused to tissues by the cooperation of light, oxygen, and a suitable sensitizing compound. The latter can arise externally, such as the hypericin ingested by cows grazing on St. Johns wort, or can be endogenous compounds, such as chlorophyll in plants and algae, or 
certain porphyrins, such as protoporphyrin IX, a normal intermediate in hemoglobin synthesis. Regardless of the source of the sensitizing pigment, if it has the appropriate chemical structure, the

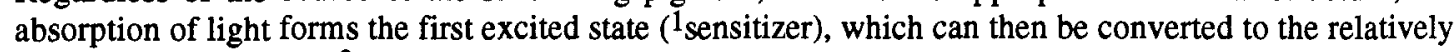
long-lived triplet state ( ${ }^{3}$ sensitizer) by the process of intersystem crossing (ISC), as seen below:

$$
\begin{aligned}
& \text { sensitizer }+h v \text {------.--> } 1_{\text {sensitizer }} \\
& 1_{\text {sensitizer }} \text { ISC }
\end{aligned}
$$

The triplet sensitizer is now capable of initiating chemical reactions that can lead to cellular damage. In the presence of oxygen another energy transfer reaction can occur, in which the excitation energy in the triplet sensitizer can be transferred to oxygen to form ${ }^{1} \mathrm{O}_{2}$, an extremely reactive and lethal form of oxygen. This reaction is shown below:

$$
{ }^{3} \text { sensitizer }+{ }^{3} \mathrm{O}_{2}-\cdots-\cdots-->>\text { sensitizer }+{ }^{1} \mathrm{O}_{2}
$$

Both the triplet sensitizer and ${ }^{1} \mathrm{O}_{2}$ can lead to damaging oxidations, known as Type $\mathrm{I}$ or Type II photochemical reactions. The ability of carotenoids to protect cells and tissues from this type of damage has been well documented, and is a result of the following two energy transfer reactions:

$$
\begin{gathered}
{ }^{3} \text { sensitizer }+ \text { carotenoid }--->\text { sensitizer }+{ }^{3} \text { carotenoid } \\
1^{1} \mathrm{O}_{2}+\text { carotenoid }-->\quad{ }^{3} \mathrm{O}_{2}+{ }^{3} \text { carotenoid }
\end{gathered}
$$

Because of the long, conjugated polyene chain in carotenoids, these molecules can undergo rotational and vibrational interactions with the solvent, and lose small quantities of energy in each of these interactions. The net result is that the energy acquired by the carotenoid from either ${ }^{3}$ sensitizer or ${ }^{1} \mathrm{O}_{2}$ is then transferred to the surrounding solvent in very small increments, and the intact, ground state carotenoid is regenerated, ready to undergo another cycle of photoprotection.

Metabolism of carotenoids to retinoids. In those animals that require vitamin A for normal growth and development, two dietary sources are available. One source is the so-called "pre-formed" vitamin A, which involves ingesting animal products that contain vitamin A. The other dietary source is the ingestion and metabolism of carotenoids than can be converted to vitamin A. Of the 600 or so carotenoids that have been described in Nature, about 50 of them have provitamin A activity (ref. 20). The most widely distributed and major source of vitamin $A$ in the diet is $B$-carotene. The original hypothesis with respect to metabolism of $B$-carotene to vitamin A (retinol) involved a hydrolytic cleavage to form 2 molecules of retinol (ref. 21). However, we now know that the reaction is more complicated, and probably involves dioxygenases that can add a molecule of oxygen across a double bond. A hypothesis was formulated by Glover that this type of reaction could occur at any of the double bonds in the polyene chain of $B$-carotene, and that the subsequent products could undergo additional chain shortening until a $\mathrm{C}_{20}$ compound, such as retinal or retinoic acid, was formed (ref. 22). This process was named excentric cleavage. However, this hypothesis was largely put aside by the observations that rat intestine and liver tissues could form retinal directly from $B$-carotene, without the appearance of any other metabolites, suggesting a central cleavage mechanism (ref. 23, 24). Even with this evidence, Ganguly and Sastry still urged retention of the excentric cleavage mechanism, based on their experiments with chicks and rats (ref. 25). More recently, Wang et al. have presented new evidence that unambiguously proves the existence of excentric cleavage in human tissues (ref. 26, 27). However, we are still left with the need to quantitate the contribution of excentric and central cleavage to overall metabolism of provitamin A carotenoids in animals.

\section{Bjological Properties: Actions and Associations}

In addition to the functions listed above, there are many biological effects which are attributed to the presence of carotenoids, either ingested through the normal diet or obtained via supplementation of the diet with physiological or pharmacological doses of carotenoids. In some cases, the causal relationship between the presence of the carotenoids and the biological action is fairly clear. In many other cases, the biological effect appears to be associated with carotenoids, but we are still in the process of establishing causality. Since we are dealing with a broad range of biological properties, I have combined the definition of actions and asociations into a single group.

Light absorption. The ability of carotenoids to act as accessory pigments in photosynthetic organisms has already been mentioned. In addition, there are many other examples in nature of the role of 
carotenoids as light absorbers. In birds and amphibians, carotenoid-containing colored oil droplets are found associated with the cone outer segments, where they serve as light filters, regulating the type of light that reaches each individual cone. In this way, the carotenoids can modulate the extent of color vision in these animals. In addition, a great deal of bird coloration is due to dietary carotenoids or their metabolites (ref. 28), and we see the same phenomenon in fish and crustaceans (ref. 29). Primates also utilize dietary carotenoids or their metabolites in the macular region of the fovea. One possible function for the accumulated lutein and zeaxanthin in the primate fovea is to attenuate blue light, and therefore increase visual acuity. However, another possibility is that they act as photoprotective pigments, protecting this sensitive region of the fovea from excess light damage. However, it is difficult to understand why nature has selected only these two xanthophyll pigments out of the multitude of pigments available in the diet or in the blood plasma.

Reproduction and fertility. There have been many reports over the years of a positive effect of dietary or supplemental carotenoids on improving fertility or reproductive capacity in a number of animals. It would seem to me that we still require additional evidence to make a firm case, and then we have to decide whether this is a carotenoid effect, or a retinoid effect.

Effects on enzymes. One very direct action of dietary or administered compounds in biological systems is the alteration of the activity of a specific enzyme. Whereas this type of information can be readily obtained in an in vitro situation, it is sometimes much more difficult to demonstrate a similar action in vivo. There are only a few examples of this type of experiment. Supplemental B-carotene has been reported to decrease the content of cytochrome P450 in mouse liver (ref. 30), and to enhance the activity of aryl hydrocarbon hydroxylase in rat intestine (ref. 31). Since both of these enzymes are important in the metabolism and detoxification of potential carcinogens, such activity of carotenoids could have significant biological effects. However, one must still determine the specificity of these effects by in vitro investigations.

Antioxidant. The ability of carotenoid pigments to act as antioxidants has been known for many years, and was usually measured by the loss of color, or "bleaching" of the carotenoid. It is only in the last 10 years that the question of mechanism of antioxidant action has been raised. The antioxidant action of carotenoids, both in vitro and in vivo, has been the subject of several recent comprehensive reviews (ref. $9,10,11$ ). Of great interest at the moment, is whether carotenoids behave as antioxidants in low-density lipoproteins (LDL), inasmuch as the oxidation of LDL is now considered to be an important causative agent in coronary heart disease (ref. 32). The results of studying carotenoid involvement in preventing LDL oxidation remain controversial. Although some investigators have reported that the addition of B-carotene to LDL in vitro results in some protection (ref. 33, 34), other workers have not been able to repeat this (ref. 35). In addition, LDL enriched with B-carotene through dietary supplementation show no difference in their responses to oxidative stress (ref. 36). So, despite the reports of a synergistic antioxidant effect between B-carotene and $\alpha$-tocopherol, both in vitro (ref. 37) and in vivo (ref. 38), we still cannot be sure of an antioxidant effect of carotenoids in LDL in vivo.

Up-regulation of Connexin 43 Gene. Several years ago, Bertram and his associates demonstrated that B-carotene and canthaxanthin could decrease the extent of malignant transformation of cells induced either by methylcholanthrene or by X-ray treatment (ref. 39). In addition, other carotenoids such as $\alpha$-carotene or lycopene were also effective (ref. 40). Lutein was inhibitory at $10 \mu \mathrm{M}$, it but appeared to increase the number of transformants at lower concentrations. $\alpha$-Tocopherol also inhibited malignant transformation, but was only about $10 \%$ as active as lycopene. This group has now shown that these carotenoids enhancegap junctional communication in $\mathrm{C} 3 \mathrm{H} / 10 \mathrm{~T} 1 / 2$ cells (ref.41), and that the molecular action appears to occur via up-regulation of the connexin 43 gene, the gene responsible for the production of one of the important components of the gap junction (ref. 42). This process was not related to the antioxidant capacities of these carotenoids, and added $\alpha$-tocopherol had no effect.

Decreased risk of degenerative diseases. The epidemiological data continues to accumulate reporting that diets rich in carotenoid-containing fruits and vegetables, or plasma levels of carotenoids, are associated with significantly decreased risks for a variety of degenerative diseases. In the case of dietary epidemiology, it is always difficult to pinpoint the exact component of the diet that may be related to the lowered risk. However, there appears to be a strong consensus among epidemiologists that the antioxidant micronutrients, such as the carotenoids, tocopherols, vitamin $\mathrm{C}$ and selenium, are the basis for reduced risk of a variety of degenerative diseases (ref. 43, 44).

Cataracts: In the United States, cataracts are a major health expense, in terms of the frequency of the routine operation that is performed to relieve this condition (ref. 45). If it were possible to prevent this condition by dietary alteration, there would be a significant improvement in the health status of many individuals, particularly the growing elderly population. In 1988, Jacques et al. published two papers indicating that people in the upper quintile for blood carotenoids had a decreased risk of cataract formation (ref. 46, 47). Since then, other epidemiological studies have supported these observations (ref. 
48,49 ) and have been reviewed recently (ref. 50,51). The largest study involving 50,828 women indicated that the only dietary nutrient that gave a lowered risk of "senile" cataract was total vitamin A intake, which included B-carotene (ref. 52). Spinach consumption was most frequently associated with lowered risk. In addition, dietary supplementation with either B-carotene or vitamin $E$ resulted in a decreased risk of cataract formation in the Royal College of Surgeons strain of rats (ref. 53).

Age-related macular degeneration: Age-related macular degeneration (ARMD) is another condition associated with aging that can lead to total blindness in otherwise healthy people. In 1988, Goldberg et al. reported a significant inverse relationship between the incidence of ARMD and the ingestion of fruits and vegetables rich in provitamin A carotenoids, such as B-carotene (ref. 54). This led to the proposal that ARMD is found in later life when antioxidant defenses are depleted (ref. 55), although Mobarhan and his associates have reported that both the extent of absorption and serum level of $B$-carotene is higher in the elderly (ref. 56). In a relatively small study, the plasma $B$-carotene level in a control group was not significantly higher than in a group of ARMD patients (ref. 57), but a more extensive study has found very significant reductions in the risk of developing neovascular ARMD as a function of plasma levels of $\alpha$-carotene, $\beta$-carotene, cryptoxanthin, lutein plus zeaxanthin, without any apparent relationship to plasma levels of lycopene, vitamin $C$, vitamin $E$ or selenium (ref. 58). Levels of antioxidants were in the highest quintile.

Coronary heart disease (CHD). This disease, in all of its ramifications, remains the major cause of death in Western societies. The etiology is complicated, although we have moved very far from simply assigning elevated cholesterol levels as the major culprit. However, the attention to cholesterol led the public to an increased concern with their diet, and many epidemiological studies have indicated that diet is an important risk factor for CHD. Several reviews have appeared either discussing the relationship between B-carotene and CHD (ref. 59), or the more general issue of antioxidants and CHD (ref. 60,61). In summary, there is epidemiological evidence for an inverse association between serum carotene levels and ischemic heart disease (ref. 62). However, there is also evidence for an inverse association between vitamin $C$ and $C H D$ (ref. 63) or vitamin $E$ and $C H D$ (ref. 64). The more recent epidemiological studies involve serum determinations, in addition to diet assessments. It may be too early to conclude whether a single antioxidant plays a role, or whether an increase in the overall "antioxidant tone" is the important factor in preventing this disease in humans.

Anticarcinogen. Following the early reports of carotenoid inhibition of tumor formation in animals supplemented with $B$-carotene (ref. 65,66 ) and the epidemiological evidence of protection against cancer by fruits and vegetables, a hypothesis was formulated that $B$-carotene might function as a dietary anticarcinogen in humans (ref. 67). The experimental protocols using animal models continue to be confounded by the use of animals that absorb dietary carotenoids only poorly, which frequently necessitates the use of pharmacological doses in order to see effects. The anticarcinogenic properties of carotenoids have been reviewed recently (ref. 10, 68, 69, 70). Most of the early work was carried out with both B-carotene and canthaxanthin, particularly in the water-dispersible beadlet preparation readily made available by Hoffmann-La Roche. Although most of the results indicated that large doses of these carotenoids could protect animals against various types of carcinogens, the results have not always been positive. Part of the problem may be exemplified by observing species differences in response to carotenoid treatment. Exposing rodents to a combined treatment with the carcinogen, dimethylbenzanthracene (DMBA) as well as the tumor promoter, phorbol myristyl acetate (PMA), results in rapid appearance of skin tumors. When Skh or Sencar mice are treated with DMBA/PMA and supplemented with $3 \%$ B-carotene in their diets, either in the form of $B$-carotene beadlets, or by adding the crystalline pigment directly to the chow, skin tumors develop. Both preparations protect Skh mice, but they do not protect the Sencar strain (ref. 71). These results emphasize the difficulty of comparing experimental results from different laboratories, for the strain differences reported above would be greatly exaggerated when one compares experiments in different species. For example, a similar protocol using DMBA/PMA-induction of skin tumors in Skh mice reported a significant decrease in the number of skin papillomas with administration of $B$-carotene at $2.4 \mathrm{mg} / \mathrm{kg}$, but no effect on the ultimate development of malignant tumors (ref. 72). Based on these observations, these authors concluded that $B$-carotene was working during the PMA-induced promotional phase of tumor formation.

In addition to $B$-carotene and canthaxanthin, other carotenoid pigments have also been reported to behave as anticarcinogens in animal studies. For example, $B$-carotene, crocetin and lycopene have all been reported to inhibit the growth of C-6 glial cells in rats (ref. 73). In addition, $\alpha$-carotene is reported to be more effective than $\beta$-carotene in preventing spontaneous liver cancer in $\mathrm{C} 3 \mathrm{H} / \mathrm{He}$ male mice, and more effective in preventing both 4-nitroquinoline-1-oxide-induced and glycerol-promoted lung cancer and DMBA-induced and PMA-promoted skin cancer in mice (ref. 74). The $\alpha$-carotene had been prepared from palm oil carotenoids, and these authors also indicated that the minor carotenoids, such as $\gamma$-carotene and lycopene, may also have activity in preventing carcinogenesis. 
Although the evidence of an association between carotenoid intake and anticarcinogenesis is not always positive, possibly due to the use of different systems in each laboratory, the majority of the results suggests a clear anticarcinogenic role of carotenoids in animal models. However, the mechanism for this effect remains unclear. In humans, the observational epidemiology has been overwhelmingly supportive of a role for dietary carotenoids in preventing lung cancer, although the evidence for other types of cancer is not as strong (ref. 75). As the large, intervention studies are nearing conclusion, we may have a much better picture in the immediate future with respect to the role of carotenoids in preventing cancer in humans.

\section{CONCLUSIONS}

There are many biological properties of carotenoids for which a causal relationship has not been adequately demonstrated. It is these properties that will deserve our increased attention in the future.

\section{ACKNOWLEDGMENTS}

The work in the author's laboratory has been supported by the National Cancer Institute, grants number R01CA 51506 and R01CA49195.

\section{REFERENCES}

1. J.A. Olson, L. Nutr. 119, 94-95 (1989).

2. P.F. Zagalsky, E.E. Eliopoulos and J.B.C. Findlay, Comp. Biochem. Physiol, 27B, 1-18 (1990).

3. R.A. Bone, J.T. Landrum and S.L. Tarsis, Vision Res. 25, 1531-1535 (1985).

4. G.J. Handelman, E.A. Dratz, C.C. Reay and F.J.G.M. van Kuijk, Invest. Ophthalmol. Vis. Sci. 29 , $850-855$ (1988).

5. C.S. Foote and R.W. Denny, L.Am. Chem. Soc, 90, 6233-6235 (1968).

6. N.I. Krinsky, in Oxygen Radicals In Chemistry And Biology (W. Bors, M. Saran and D. Tait, Eds.) pp. 453-464, Walter de Gruyter \& Co., Berlin. (1984).

7. P. Di Mascio, S. Kaiser and H. Sies, Arch. Biochem. Biophys. 274, 532-538 (1989).

8. P.F. Conn, Schalch and T.G. Truscott, J. Photochem. Photobiol. B: Biol 11, $41-47$ (1991).

9. N.I. Krinsky, Eree Radic. Biol. Med. 2, 617-635 (1989).

10. E.J. Rousseau, A.J. Davison and B. Dunn, Eree Radic. Biol. Med. 12, 407-433 (1992).

11. P. Palozza and N.I. Krinsky, Meth. Enzymol. 213, 403-420 (1992).

12. G.J. Handelman, F.J.G.M. van Kuijk, A. Chatterjee and N.I. Krinsky, Eree Radic. Biol. Med. 10, 427-437 (1991).

13. T.A. Kennedy and D.C. Liebler, Chem. Res. Toxicol, 4, 290-295 (1991).

14. R.C. Mordi, J.C. Walton, G.W. Burton, L. Hughes, K.U. Ingold, D.A. Lindsay and D.J. Moffatt, Tetrahedron 49, 911-928 (1993).

15. D.S. Goodman and J.A. Olson, Meth. Enzymol. 15, 462-475 (1968).

16. G. Wald, P.K. Brown, R. Hubbard and W. Oroshnik, Proc. Natl. Acad. Sci. USA 41, 438-451 (1955).

17. A. Ben-Amotz, A. Lers and M. Avron, Plant Physiol 86, 1286-1291 (1988).

18. Y. Koyama, Photochem. Photobiol 53, 65S-66S (1991).

19. R.J. Cogdell, Pure \& Apol. Chem this volume.

20. H. Pfander, Ed., Key to Carotenoids, pp. 1-296, Birkhäuser: Basel, 1987.

21. P. Karrer, R. Morf and K. Schöpp, Helv, Chim. Acta 14, 1431-1436 (1931).

22. J. Glover, in Vitamins and Hormones (R.S. Harris and D.J. Ingle, Eds.) pp. 371-386, Academic Press, New York and London. vol 18 (1960).

23. J.A. Olson and O. Hayaishi, Proc. Natl. Acad. Sci. USA 54, 1364-1370 (1965).

24. D.S. Goodman and H.S. Huang, Science 149, 879-880 (1965).

25. J. Ganguly and P.S. Sastry, World. Rev. Nutr. Diet. 45, 198-220 (1985).

26. X.-D. Wang, G.-W. Tang, J.G. Fox, N.I. Krinsky and R.M. Russell, Arch. Biochem. Biophys. 285, 8-16 (1991).

27. X.-D. Wang, N.I. Krinsky, G. Tang and R.M. Russell, Arch. Biochem. Biophys. 293, 293-304 (1992).

28. A.H. Brush, EASEB J 4, 2969-2977 (1990).

29. S. Liaaen-Jensen, Pure \& Appl. Chem. 63, 1-12 (1991).

30. T.K. Basu, N.J. Temple and J. Ng, L. Clin. Biochem. Nutr, 3, 95-102 (1987).

31. T.E. Edes, W. Thornton Jr. and J. Shah, J. Nutr. 119, 796-799 (1989).

32. H. Esterbauer, J. Gebicki, H. Puhl and G. Jürgens, Eree Radic. Biol. Med. 13, 341-390 (1992).

33. I. Jialal, E.P. Norkus, L. Cristol and S.M. Grundy, Biochim. Biophys. Acta 1086, 134-138 (1991).

34. M. Naruszewicz, E. Selinger and J. Davignon, Metabolism 41, 1215-1224 (1992). 
35. J.M. Gaziano, B. Frei, P.R. Ridker, N.I. Krinsky, E.J. Johnson, J.E. Buring, M.J. Stampfer and C.H. Hennekens, Circulation 88 (Suppl.11), in press (1993).

36. P.D. Reaven, A. Khouw, W.F. Beltz, S. Parthasarathy and J.L. Witztum, Arterioscler. Thromb. 13, 590-600 (1993).

37. P. Palozza and N.I. Krinsky, Arch. Biochem. Biophys. 297, 184-187 (1992).

38. B. Leibovitz, M.-L. Hu and A.L. Tappel, L Nutr. 120, 97-104 (1990).

39. A.O. Pung, J.E. Rundhaug, C.N. Yoshizawa and J.S. Bertram, Carcinogenesis 9, 1533-1539 (1988).

40. J.S. Bertram, A. Pung, M. Churley, T.J. Kappock IV , L.R. Wilkins and R.V. Cooney, Carcinogenesis 12, 671-678 (1991).

41. L.-X. Zhang, R.V. Cooney and J.S. Bertram, Carcinogenesis 12, 2109-2114 (1991).

42. L.-X. Zhang, R.V. Cooney and J.S. Bertram, Cancer Res, 52, 5707-5712 (1992).

43. B.N. Ames, Science 221, 1256-1264 (1983).

44. K.F. Gey, Biochem. Soc. Trans. 18, 1041-1045 (1990).

45. A. Taylor, Ann. New York Acad. Sci. 669, 111-124 (1992).

46. P.F. Jacques, S.C. Hartz, L.T. Chylack, R.B. McGandy and J.A. Sadowski, Am. J. Clin. Nutr. 48 , $152-158$ (1988).

47. P.F. Jacques, L.T. Chylack, R.B. McGandy and S.C. Hartz, Arch. Ophthalmol, 106, 337-340 (1988).

48. M.C. Leske, L.T. Chylack and S.-Y. Wu, Arch. Ophthalmol 109, 244-251 (1991).

49. P. Knekt, M. Heliovaara, A. Rissanen, A. Aromaa and R.-K. Aaran, Br. Med. J 305, 1392-1394 (1992).

50. P.F. Jacques and A. Taylor, in Micronutrients in Health and Disease Prevention. (A. Bendich and C.E. Butterworth Jr., Eds.) pp. 359-379, Marcel Dekker, New York. (1991).

51. A. Taylor, L.Am. Coll. Nutr. 12, 138-146 (1993).

52. S.E. Hankinson, M.J. Stampfer, J.M. Seddon, G.A. Colditz, B.N. Rosner, F.E. Speizer and W.C. Willett, BMJ 305, 335-339 (1992).

53. H.H. Hess and J.S.J. Zigler, Invest. Ophthalmol. Vis. Sci. 32, 1100 (1991).

54. J. Goldberg, G. Flowerdew, E. Smith, J.A. Brody and M.O.M. Tso, Am. J.Epidemiol, 128, 700-710 (1988).

55. H. Taylor, B. Muñoz, S. West, N.M. Bressler, S.B. Bressler and F.S. Rosenthal, Tr. Am. Ophth. Soc. 88, 163-178 (1991).

56. S.B. Sugarman, S. Mobarhan, P.E. Bowen, M. Stacewicz-Sapuntzakis, P. Langenberg, C. Henderson, R. Kiani, H. Friedman and D. Lucchesi, J. Am. Coll. Nutr. 10, 297-307 (1991).

57. C.D. Drews, P. Sternberg, P.S. Samiec, D.P. Jones, R.L. Reed, E. Flagg, A. Boddie and R. Tinkelman, Invest. Ophthalmol. Vis. Sci. 34, 1158 (1993).

58. E.D.C.-C.S. Group, Arch. Ophthalmol. 111, 104-109 (1993).

59. H. Gerster, Internat. J. Vit. Nutr. Res. $61,277-291$ (1991).

60. J.M. Gaziano, J.E. Manson, J.E. Buring and C.H. Hennekens, Ann. N. Y. Acad. Sci. 669, 249-259 (1992).

61. D. Kritchevsky, Nutr. Today 27, 30-33 (1992).

62. H.B. Stähelin, M. Eichholzer and K.F. Gey, Bibl. Nutr. Dieta 49, 24-35 (1992).

63. K.F. Gey, U.K. Moser, P. Jordan, S. H.B., M. Eichholzer and E. Lüdin, Am. J. Clin, Nutr. 57, 787S-797S (1993).

64. E.B. Rimm, M.J. Stampfer, A. Ascherio, E. Giovannucci, G.A. Colditz and W.C. Willett, New Engl. I. Med. 328, 1450-1456 (1993).

65. M.M. Mathews-Roth, in Current Chemotherapy and Infectious Diseases (J.D. Nelson and C. Grassi, Eds.) pp. 1503-1505, Am. Soc. Microbiol., Washington D.C. (1980).

66. L. Santamaria, A. Bianchi, A. Arnaboldi and L. Adreoni, Boll. Chim. Farm. 119, 745-748 (1980).

67. R. Peto, R.J. Doll, J.D. Buckley and M.B. Sporn, Nature 290, 201-208 (1981).

68. N.I. Krinsky, in Eree Radicals and Aging (I. Emerit and B. Chance, Eds.) pp. 227-234, Birkhäuser, Basel. (1992).

69. N.I. Krinsky, in Vitamins and Cancer Prevention (G.A. Bray and D.H. Ryan, Eds.) pp. 260-270, Louisiana State Univ. Press, Baton Rouge. (1993).

70. N.I. Krinsky, Ann. Rev, Nutr. 13, 561-587 (1993).

71. L.A. Lambert, W.H. Koch, W.G. Wamer and A. Kornhauser, Nutr. Cancer 13, 213-221 (1990).

72. H.H. Steinel and R.S.U. Baker, Cancer Lett. 51, 163-168 (1990).

73. C.-J. Wang, M.-Y. Chou and J.-K. Lin, Cancer Letters 48, 135-142 (1989).

74. M. Murakoshi, J. Takayasu, O. Kimura, E. Kohmura, H. Nishino, A. Iwashima, J. Okuzumi T. Sakai, T. Sugimoto, J. Imanishi and R. Iwasaki, J. Natl, Cancer Inst. 81, 1649-1652 (1989).

75. R.G. Ziegler, Am. J. Clin. Nutr. 53, 251S-259S (1991). 